\title{
PAIR PRODUCTION DENSITY IN STRONG QED
}

\author{
SANG PYO KIM \\ Department of Physics, Kunsan National University, Kunsan 573-701, Korea \\ Asia Pacific Center for Theoretical Physics, Pohang 790-784, Korea \\ sangkim@kunsan.ac.kr \\ HYUN KYU LEE \\ Department of Physics, Hanyang University, Seoul 133-791, Korea \\ hyunkyu@hanyang.ac.kr \\ YONGSUNG YOON \\ Department of Physics, Hanyang University, Seoul 133-791, Korea \\ cem@hanyang.ac.kr
}

\begin{abstract}
The current status of the research on pair production in strong QED is briefly reviewed not only for strong electric fields but also for strong magnetic fields in magnetically dominated astrophysical environment. A particular attention is paid to the spectral production number in the in- and out-state formalism and a possible prescription of the momentum integration is proposed to obtain the production density.
\end{abstract}

Keywords: Strong QED; vacuum instability; pair production density; finite temperature effect.

PACS Nos.: 12.20.-m, 13.40.-f, 12.20.Ds, 11.15.Tk

\section{Introduction}

The instability of vacuum in a strong electromagnetic field against production of charged particles that couple to the field has a long history of investigations. Sauter, Heisenberg and Euler, and Weisskopf studied the effective action, ${ }^{1}$ and Schwinger systematically derived the effective action in a gauge invariant form in a constant electromagnetic field and calculated the pair-production rate, the so-called Schwinger process. ${ }^{2}$ There have been since then many attempts to find the effective actions in various configurations of electromagnetic fields (for a review, see Ref. 3).

Computing the effective action in nontrivial electric field configurations has been a challenging problem due to the vacuum instability; however, it has recently become one of the interesting subjects partly because direct tests of strong QED are possible in terrestrial experiments in the near future ${ }^{4}$ and partly because strong QED can be applied to astrophysical objects with strong electromagnetic fields beyond the critical strength. 
For example, the explosive phenomena, such as gamma ray bursts and magnetars, indicate very strong magnetic fields ${ }^{5}$ around the compact objects that are considered responsible for powering the explosive events. The inferred field strengths might be as large as $\sim 10^{15} \mathrm{G}$ or even larger. ${ }^{6}$ It has been accepted that strong magnetic fields in astrophysical environments are stable against pair production of charged particles through minimal interactions. However, two (H.K.L. and Y.Y.) of authors have recently proposed neutrino pair production in strong magnetic field backgrounds, provided that massive neutrinos couple to the electromagnetic field through the Pauli coupling with a magnetic moment. ${ }^{7}$

On the other hand, in quantum field theory for strong QED, two (S.P.K. and H.K.L.) of the authors have introduced the evolution operator method for computing the effective action in scalar QED. The evolution operator evolves the ingoing vacuum to the outgoing vacuum in the time-dependent gauge and consists of two parts, a phase part and a squeeze operator, whose parameters are determined by the Bogoliubov coefficients. ${ }^{8}$ The effective action is then defined as the scattering amplitude between the ingoing and outgoing vacua a la Schwinger's action principle for the in- and out-state formalism. ${ }^{8,9,10,11}$ The renormalized effective action for a constant electric field, given by a Bogoliubov coefficient for each momentum, correctly yields not only the vacuum polarization ${ }^{2}$ but also the vacuum persistence. ${ }^{12,13} \mathrm{We}$ have further elaborated the in- and out-state state formalism and found the exact one-loop effective action for the Sauter-type electric field, $E_{0} \operatorname{sech}^{2}(t / \tau)$, with a proper renormalization procedure. ${ }^{14}$

In the in- and out-state formalism, the imaginary part of the effective action in scalar and spinor QED satisfies the general relations between the vacuum persistence and the total mean number of produced scalars and fermions. ${ }^{13,14}$ The pair-production rate from the imaginary part may be compared to the earlier works for inhomogeneous electromagnetic fields, which have developed the uniform semiclassical approximation, ${ }^{15}$ the WKB action in the phase-integral ${ }^{16}$ or the WKB approximation $^{17}$ and the worldline instanton method $^{18}$ for the mean number at each momentum, $\mathcal{N}_{\mathbf{k}}=\left|\nu_{\mathbf{k}, \omega}\right|^{2}$, that is, the absolute square of the Bogoliubov coefficient. The production-rate density of particle pairs, $\mathcal{R}$, which is physically more interesting and can be measured in experimentations, is given by the momentum integration of the mean number. However, the $k_{z}$-integral is not trivial in this method; it diverges in a constant electric field because $\mathcal{N}_{\mathbf{k}}$ is independent of $k_{z}$, and it is complicated to get an analytical expression in the Sauter-type field configuration.

In this paper for Proceedings we propose a prescription how the momentum integration can be done properly at zero and finite temperature. The organization of this paper is as follows. In Section 2, we briefly review the neutrino pair production via the Pauli coupling in strong magnetic fields, and in Section 3, the spectral production density in the in- and out-state formalism for scalar and spinor QED. In Section 4, a prescription is proposed for the momentum integration to get the production-rate density of charged particle pairs both for a constant electric field and for the Sauter-type electric field. 


\section{Neutrino Pair Production in Strong Magnetic Field through Pauli Coupling}

The very strong magnetic fields ${ }^{5}$ around compact astrophysical objects, whose strength has been inferred as large as $B \sim 10^{15} \mathrm{G}$ or even larger, ${ }^{6}$ may be the source for the explosive phenomena, such as gamma ray bursts and magnetars. Since most of astrophysical environments are electrically neutral except for a very narrow region near the surface of compact objects, electric fields are always accompanied by magnetic fields. The rotation of the accompanying magnetic field induces an electric field $E \sim B v_{\text {rotation. }}$. For $B \sim 10^{15} \mathrm{G}$, the induced electric field is estimated to be order of $E \sim 10^{17} \mathrm{~V} / \mathrm{cm}$, which is at least one order larger than the critical field strength for electro-positron pair production via the Schwinger process. However, the magnetosphere for this configuration is dominated by the magnetic field, $\mathcal{F}=\left(B^{2}-E^{2}\right) / 2>0$, and thus does not lead to production of charged particle pairs in scalar and spinor QED, ${ }^{16,15}$ which is a purely electric effect. Hence the strong magnetic fields in astrophysical environments have been regarded stable against production of charged particles through the minimal interaction only.

Though the minimal coupling rooted on the local gauge invariance of the QED action is of fundamental nature, theories with non-minimal couplings have also been proposed as an effective theory. Pauli introduced a non-minimal coupling of spin-1/2 fermions with electromagnetic fields, which could be interpreted as an effective interaction of fermions with an anomalous magnetic moment. ${ }^{19,20}$ For neutral fermions with non-vanishing magnetic moments, it is the Pauli term through which the electromagnetic interactions can be probed.

Recently, a possible scenario has been proposed for production of neutral Dirac fermion pairs in the magnetic fields stronger than the critical field strength $\left(B_{c}=m / \mu\right),{ }^{7}$ which is the ratio of the fermion mass to its magnetic moment. This implies that magnetic field configurations beyond the critical field strength become unstable, and the pair production can occur as a purely magnetic effect. It has also been investigated that neutrinos may be Majorana fermions with a transition magnetic moment. It is found that a nonzero imaginary part of the effective potential develops when the magnetic fields are stronger than the critical field strength $B_{c}\left(\approx m_{\nu} / \mu_{\nu}\right)$, similarly to the case of neutral Dirac fermions with a diagonal magnetic moment. ${ }^{7}$ This implies that strong magnetic fields may be unstable beyond the critical field strength and produce the neutrino pairs with the rate density $\sim\left(m_{\nu}^{4} c^{5} / \hbar^{4}\right)\left(B / B_{c}\right)^{4}$. All these suggest that the compact astrophysical object with the strong magnetic field configurations beyond the critical field strength may be a source of continuous production of neutrino pairs through the magnetic production mechanism.

The physical origin of the instability in the Pauli interaction can be traced back to the ground state that crosses the zero energy state under a strong enough 
magnetic field. ${ }^{\mathrm{a}}$ A similar instability due to the magnetic coupling has been observed for the massive gauge bosons in Ref. 11.

\section{Vacuum Persistence and Spectral Production Number}

In the in- and out-state formalism adopted from Ref. 14, the spectral production rate of the charged particles can be calculated from the imaginary part of the effective action, as will be briefly reviewed below. In the time-dependent gauge, an electric field along the $z$-direction is given by $E(t)=-\partial A_{z}(t) / \partial t$. The Fourier component of the Klein-Gordon equation for scalar QED and the spin-diagonal component of the Dirac equation for spinor QED satisfy [in units with $\hbar=c=1$ and with metric signature $(+,-,-,-)]$

$$
\left[\partial_{t}^{2}+m^{2}+\mathbf{k}_{\perp}^{2}+\left(k_{z}+q A_{z}(t)\right)^{2}+2 i \sigma q E(t)\right] \phi_{\mathbf{k} \sigma}(t)=0,
$$

where $\sigma=0$ for scalar particles and $\sigma= \pm 1 / 2$ for spin- $1 / 2$ fermions.

First, for a constant electric field, $A_{z}=-E t$, the equation describes the scattering problem over an inverted harmonic potential, $-\left(q E t-k_{z}\right)^{2}$, and a complex energy, $m^{2}+\mathbf{k}_{\perp}^{2}+2 i \sigma q E$. Using the method in Ref. 14, the effective Lagrangian per unit volume for spinor QED is given by

$$
\mathcal{L}_{\mathrm{eff}}^{\mathrm{sp}}=-i \frac{1}{2} \frac{q E}{2 \pi} \int \frac{d^{2} \mathbf{k}_{\perp}}{(2 \pi)^{2}} \int_{0}^{\infty} \frac{d s}{s} e^{i \frac{m^{2}+\mathbf{k}_{\perp}^{2}}{2 q E} s}\left[\operatorname{coth}(s / 2)-\frac{2}{s}+\frac{s}{12}\right] .
$$

The prefactor $q E / 2 \pi$ in Eq. (2) comes from the $k_{z}$-momentum integral, ${ }^{10}$ which will be discussed in detail in Section 4. Integrating over the momentum and doing a contour integral in a complex plane to rotate the integral to the real proper time, the renormalized effective action is given by

$$
\begin{aligned}
\mathcal{L}_{\mathrm{eff}}^{\mathrm{sp}}= & \frac{1}{8 \pi^{2}} \mathcal{P} \int_{0}^{\infty} \frac{d s}{s^{3}} e^{-m^{2} s}\left[(q E s) \cot (q E s)-\frac{1}{s}+\frac{(e E s)^{3}}{3}\right] \\
& +i \frac{(q E)^{2}}{8 \pi^{3}} \sum_{n=1}^{\infty} \frac{1}{n^{2}} e^{-\frac{\pi m^{2} n}{q E}}
\end{aligned}
$$

which agrees with the exact result in the proper time integral by Schwinger. ${ }^{2}$

Second, for a Sauter-type electric field, $E(t)=E_{0} \operatorname{sech}^{2}(t / \tau)$, which effectively acts for a finite period of time, one may choose the gauge as

$$
A_{\mu}=\left(0,0,0,-E_{0} \tau\left(1+\tanh \left(\frac{t}{\tau}\right)\right)\right),
$$

and obtain the vacuum persistence, twice of the imaginary part of the effective potential, as ${ }^{14}$

$$
2\left(\operatorname{Im} \mathcal{L}_{\text {eff }}\right)= \pm(2 S+1) \int \frac{d^{3} \mathbf{k}}{(2 \pi)^{3}} \ln \left(1 \pm \mathcal{N}_{\mathbf{k}}\right)
$$

\footnotetext{
${ }^{a}$ One must be careful in deriving the effective potential particularly to get an imaginary part, because the integration over the determinant of Hamiltonian involves zero's. ${ }^{7}$
} 
Here, $2 S+1$ is the spin multiplicity and the upper (lower) sign is for scalar particles (spin-1/2 fermions), respectively. The spectral production number of produced pairs is given by

$$
\mathcal{N}_{\mathbf{k}}=\frac{\cosh (2 \pi \lambda) \pm \cosh \left(\pi \tau \omega_{\mathbf{k}, \text { out }}-\pi \tau \omega_{\mathbf{k}, \text { in }}\right)}{2 \sinh \left(\pi \tau \omega_{\mathbf{k}, \text { in }}\right) \sinh \left(\pi \tau \omega_{\mathbf{k}, \text { out }}\right)} .
$$

where $\lambda=\sqrt{\left(q E_{0} \tau^{2}\right)^{2}-(1-2|\sigma|) / 4}$, and $\omega_{\mathbf{k}, \text { in }}$ and $\omega_{\mathbf{k}, \text { out }}$ denote the initial frequency at $t=-\infty$ and the final frequency at $t=+\infty$, respectively. The production density of particle pairs from the vacuum, $\mathcal{N}$, can be obtained by integrating $\mathcal{N}_{\mathbf{k}}$ over the momentum space, as will be shown below.

\section{Momentum Integrations of Spectral Production Number}

In the in- and out-state formalism, the mean number of produced particle pairs at zero temperature at each momentum is the absolute square of the Bogoliubov coefficient, $\mathcal{N}_{\mathbf{k}}=\left|\nu_{\mathbf{k}, \omega}\right|^{2}$. For a constant electric field along the $z$-direction, $\nu_{\mathbf{k}, \omega}$ is obtained as ${ }^{14}$

$$
\nu_{\mathbf{k}, \omega}=e^{-i p \pi} e^{i \omega \frac{k_{z}}{q E}}, \quad p=-\frac{1}{2}-i \frac{m^{2}+\mathbf{k}_{\perp}^{2}+2 i \sigma q E}{2 q E},
$$

where the frequency $\omega$ is determined by $\mathbf{k}$ at on-shell from the dispersion relation of the particle. The extra phase factor, $e^{i \omega k_{z} / q E}$, of the Bogoliubov coefficient comes from the time shift $t=t^{\prime}+k_{z} / q E$ with the ingoing (outgoing) vacuum at $t^{\prime}=$ $-\infty(+\infty)$, which is introduced to regulate the production-rate density of particle pairs. The vacuum becomes unstable in strong electric fields and pairs of charged particles are created from the Dirac sea via quantum tunneling.

The production-rate density of particle pairs, $\mathcal{R}$, is given by the momentum integration of the mean number of produced particle pairs. However, there is a caveat in doing this, since $\mathcal{N}_{\mathbf{k}}$ is independent of $k_{z}$ and the $k_{z}$-integration thus diverges. A common practice is to restrict the momentum integration to the transverse directions $\mathbf{k}_{\perp}$ and multiply the density of state $q E / 2 \pi$ for the longitudinal direction, in an analogy with the magnetic field case. ${ }^{10}$ Alternatively, we may regularize the divergence, using the extra phase factor in Eq. (7) and integrating the energy-momentum at off-shell instead of on-shell. In other words, we take $\nu_{\mathbf{k}, \omega^{\prime}}^{*} \nu_{\mathbf{k}, \omega}$ at off-shell and integrate over $\omega^{\prime}$ and the three-momentum $\mathbf{k}$ as

$$
\begin{aligned}
\mathcal{R} & =\int \frac{d \omega^{\prime}}{2 \pi} \frac{d^{3} \mathbf{k}}{(2 \pi)^{3}} \nu_{\mathbf{k}, \omega^{\prime}}^{*} \nu_{\mathbf{k}, \omega}=\int \frac{d \omega^{\prime}}{2 \pi} \frac{d k_{z}}{2 \pi} \frac{d^{2} \mathbf{k}_{\perp}}{(2 \pi)^{2}} e^{i\left(\omega-\omega^{\prime}\right) \frac{k z}{q E}} e^{-\frac{\pi\left(m^{2}+\mathbf{k}_{\perp}^{2}\right)}{q E}} \\
& =\frac{(q E)^{2}}{8 \pi^{3}} e^{-\frac{\pi m^{2}}{q E}} .
\end{aligned}
$$

The density of pairs is consistent with the Schwinger's result obtained from the imaginary part of the effective action. This regularization can be also used in calculating the production-rate density of particle pairs at finite temperature.

Next, we consider a pulselike electric field of Sauter-type, $A_{z}(t)=-E_{0} \tau(1+$ $\tanh (t / \tau))$. The initial state is the Minkowski vacuum because the gauge field is 
zero. The equation of motion is exactly solvable, and the Bogoliubov coefficients are known. At zero temperature, the spectral production number of particle pairs for a sufficiently long duration $(\tau \gg 1 / \sqrt{\pi q E})$ is approximately given in the phaseintegral method ${ }^{16}$

$$
\mathcal{N}_{\mathbf{k}} \cong e^{-\frac{\pi m^{2}}{q E}-\frac{\pi \mathbf{k}_{\perp}^{2}}{q E}-\frac{\pi m^{2}}{q E} \frac{k_{z}^{2}}{(q E \tau)^{2}}}
$$

The exponent is the expansion of the leading term of the exact mean number up to quadratic order in momenta and gives the worldline instanton result. ${ }^{18}$ Then the production density of particle pairs is

$$
\mathcal{N}=\int \frac{d k_{z}}{2 \pi} \frac{d^{2} \mathbf{k}_{\perp}}{(2 \pi)^{2}} e^{-\frac{\pi m^{2}}{q E} \frac{k_{z}^{2}}{(q E \tau)^{2}}} e^{-\frac{\pi m^{2}}{q E}-\frac{\pi \mathbf{k}_{\perp}^{2}}{q E}} \cong \frac{(q E)^{5 / 2} \tau}{8 \pi^{3} m} e^{-\frac{\pi m^{2}}{q E}}
$$

Comparing to the result (8) for the constant electric field, the result for Sauter-type field is $\mathcal{N} / \tau \approx \sqrt{q E} \mathcal{R} / m$, which has an additional factor $\sqrt{q E} / m$, suppressing pair production for electric field weaker than the critical strength.

\section{Spectral Production Number at Finite Temperature}

At finite temperature, instead of the charge neutral vacuum, we should consider an initial thermal (in general, non-neutral) ensemble of particles at $T$. Considering no mode-mixing among different momenta and taking into account the vacuum pair production, the induced pair production and the stimulated pair annihilation, the mean number at each momentum is factorized into the mean number at zero temperature and the initial thermal distribution factor $\left(1 \pm 2 f_{B / F}\right)$, where $f_{B / F}$ is the Bose-Einstein or Fermi-Dirac distribution function. ${ }^{8,21}$ The spectral number density of produced pairs at finite temperature will be studied for a constant electric field and a pulselike electric field of Sauter-type.

First, in the constant electric field background, the concept of the initial thermal ensemble is not obvious, because the Schwinger process persists from the beginning and makes the initial thermal ensemble unstable. However, we may consider a free initial thermal ensemble at temperature $T$ without the electric field. The initial ensemble may not be spoiled abruptly, provided that the constant electric field strength is weaker than the critical strength. Thus, the production-rate density of particle pairs at finite temperature is

$$
\begin{aligned}
\mathcal{R}(T) & =\int \frac{d \omega^{\prime}}{2 \pi} \frac{d^{3} \mathbf{k}}{(2 \pi)^{3}} e^{i\left(\omega-\omega^{\prime}\right) \frac{k z}{q E}} e^{-\frac{\pi\left(m^{2}+\mathbf{k}_{\perp}^{2}\right)}{q E}}\left(1 \pm 2 f_{B / F}\left(\frac{\sqrt{m^{2}+\mathbf{k}_{\perp}^{2}+k_{z}^{2}}}{k_{B} T}\right)\right) \\
& =\frac{(q E)^{2}}{8 \pi^{3}} e^{-\frac{\pi m^{2}}{q E}} \pm \frac{q E}{\pi} \int_{m}^{\infty} \frac{d \xi}{2 \pi} \xi e^{-\frac{\pi \xi^{2}}{q E}} f_{B / F}\left(\frac{\xi}{k_{B} T}\right)
\end{aligned}
$$

where we have assumed the interaction-free initial distribution, and $+(-)$ is for bosons (fermions). The first term is a pure quantum effect at zero temperature while the second term is a consequence of thermal and quantum effects. 
For a thermal energy lower than the rest mass energy, approximating the thermal distribution as the Boltzmann distribution $f_{B / F}\left(\xi / k_{B} T\right) \cong e^{-\xi / k_{B} T}$, the production-rate density at temperature $T$ is given by

$$
\mathcal{R}(T) \simeq \mathcal{R} \pm \frac{(q E)^{2}}{4 \pi^{3}} e^{-\frac{\pi m^{2}}{q E}-\frac{m}{k_{B} T}} \mp \frac{(q E)^{5 / 2}}{8 \pi^{3} k_{B} T}\left(1-\operatorname{erf}\left(\xi_{0}\right)\right) e^{\frac{q E}{4 \pi\left(k_{B} T\right)^{2}}}
$$

where $\xi_{0}=\sqrt{\pi m^{2} / q E}+\sqrt{q E / 4 \pi\left(k_{B} T\right)^{2}}$. For $\xi_{0} \gg 1$, that is, the electric field weaker than the critical strength or temperature lower than the field strength, approximating the error function as $\operatorname{erf}\left(\xi_{0}\right) \simeq 1-e^{-\xi_{0}^{2}} / \sqrt{\pi} \xi_{0}$, we get

$$
\mathcal{R}(T) \simeq \mathcal{R} \pm \frac{(q E)^{2}}{2 \pi^{2}} \frac{m k_{B} T}{q E+2 \pi m k_{B} T} e^{-\frac{\pi m^{2}}{q E}-\frac{m}{k_{B} T}}
$$

where the first term is the zero temperature result and the second term is the effect of the initial thermal ensemble. As expected, the thermal effect enhances the boson pair production but suppresses fermion pair production due to the Pauli exclusion principle.

For the Sauter-type electric field, we may consider an initial thermal ensemble, which is almost free from the Schwinger process, since the interaction is completely negligible in the far past. Multiplying the interaction-free thermal factor similarly, the production-rate density of particle pairs at temperature $T$ is

$$
\begin{aligned}
\mathcal{N}(T) & \simeq \mathcal{N} \pm 2 \int \frac{d k_{z}}{2 \pi} \frac{d^{2} \mathbf{k}_{\perp}}{(2 \pi)^{2}} e^{-\frac{\pi m^{2}}{q E} \frac{k_{z}^{2}}{(q E \tau)^{2}}} e^{-\frac{\pi m^{2}}{q E}-\frac{\pi \mathbf{k}_{\perp}^{2}}{q E}} e^{-\frac{m}{k_{B} T}-\frac{\mathbf{k}_{\perp}^{2}}{2 m k_{B} T}-\frac{k_{z}^{2}}{2 m k_{B} T}} \\
& =\mathcal{N} \pm \frac{(q E)^{5 / 2} \tau\left(k_{B} T\right)}{\left(2 \pi^{2}\right)\left(q E+2 \pi m k_{B} T\right)} \sqrt{\frac{2 \pi m^{3} k_{B} T}{2 \pi m^{3} k_{B} T+q E(q E \tau)^{2}}} e^{-\frac{\pi m^{2}}{q E}-\frac{m}{k_{B} T}}
\end{aligned}
$$

where the thermal distribution approximated by the Boltzmann distribution has been expanded up to the quadratic order in the momenta in the exponent. It is interesting to note that at high temperature, such that $m^{3} k_{B} T \gg q E(q E \tau)^{2}$, the finite temperature result (14) for Sauter-type electric field reduces to $\mathcal{N}(T) / \tau \approx$ $\sqrt{q E} \mathcal{R}(T) / m$, which has the same additional factor $\sqrt{q E} / m$, when compared to Eq. (13) for the constant electric field, whose behavior also holds at zero temperature.

Note added in proof: After this paper was submitted, the in- and out-state formalism for the effective action was extended to spatially localized electric fields, ${ }^{22}$ and a novel method was also proposed for the effective action at finite temperature, where the leading term of the imaginary part gives the production-rate density (13) as for the case of zero temperature. ${ }^{23}$

\section{Acknowledgments}

The work of S. P. K. was supported by the Korea Research Foundation Grant funded by the Korean Government (MOEHRD) (KRF-2007-C00167) and the work of H. K. L. was supported by the Korea Science and Engineering Foundation (KOSEF) grant funded by the Korea government (MOST) (No. R01-2006-00010651-0). 


\section{References}

1. F. Sauter, Z. Phys. 69, 742 (1931); W. Heisenberg and H. Euler, Z. Physik 98, 714 (1936); V. Weisskopf, K. Dan. Vidensk. Selsk. Mat. Fys. Medd. 14, 6 (1936).

2. J. Schwinger, Phys. Rev. 82, 664 (1951).

3. G. V. Dunne, "Heisenberg-Euler Effective Lagrangians: Basics and Extensions," in From Fields to Strings: Circumnavigating Theoretical Physics, edited by M. Shifman, A. Vainshtein, and J. Wheater, (World Scientific, Singapore, 2005), Vol. I, pp. 445-522 [arXiv:hep-th/0406216].

4. A. Ringwald, Phys. Lett. B 510, 107 (2001); "Fundamental Physics at an X-Ray Free Electron Laser," in Electromagnetic Probes of Fundamental Physics, edited by W. Marciano and S. White (World Scientific, Singapore, 2003), pp. 63-74 [arXiv:hepph/0112254]; "Boiling the Vacuum with an X-Ray Free Electron Laser," in Quantum Aspects of Beam Physics 2003, edited by P. Chen and K. Reil (World Scientific, Singapore, 2004), pp. 149-163 [hep-ph/0304139].

5. P. M. Woods and C. Thomson, "Soft Gamma Repeaters and Anomalous X-ray Pulsars: Magnetar Candidates," [arXiv:astro-ph/0406133].

6. V. V. Usov, Nature 357, 472 (1992); C. Thomson and R. C. Duncan, Astrophs. J. 408, 194 (1993); J. I. Katz, Astrophys. J. 490, 633 (1997); M. A. Ruderman, L. Tao and W. Kluzniak, Astrophys. J. 542, 243 (2000).

7. H. K. Lee and Y. Yoon, JHEP 03 (2006) 78; JHEP 03 (2007) 86; Mod. Phys. Lett. A 22, 2081 (2007).

8. S. P. Kim and H. K. Lee, J. Korean Phys. Soc. 54, 2604 [arXiv: 0806.2496].

9. J. Schwinger, Proc. Nat. Acad. Sci. 37, 452 (1951); B. S. DeWitt, Phys. Rep. 19, 295 (1975); A. I. Nikishov, JETP 96, 180 (2003).

10. A. I. Nikishov, Zh. Eksp. Teor. Fiz. 57, 1210 (1970) [Sov. Phys. JETP 30, 660 (1970)].

11. J. Ambjorn, R. J. Hughes, and N. K. Nielsen, Ann. Phys. 150, 92 (1983)

12. E. S. Fradkin, D. M. Gitman, and S. M. Shvartsman, Quantum Electrodynamics with Unstable Vacuum (Springer-Verlag, Berlin, 1991); S. P. Gavrilov, D. M. Gitman, and J. L. Tomazelli, Nucl. Phys. B 795, 645 (2008).

13. W-Y. P. Hwang and S. P. Kim, Phys. Rev. D 80, 065004 (2009).

14. S. P. Kim, H. K. Lee, and Y. Yoon, Phys. Rev. D 78, 105013 (2008).

15. G. Dunne and T. M. Hall, Phys. Lett. B 419, 322 (1998); Phys. Rev. D 60, 065002 (1999).

16. S. P. Kim and D. N. Page, Phys. Rev. D 65, 105002 (2002); Phys. Rev. D 73, 065020 (2006); Phys. Rev. D 75, 045013 (2007); S. P. Kim, AIP Conf. Proc. 1150, 95 [arXiv:0902.3486].

17. H. Kleinert, R. Ruffini, and S-S. Xue, Phys. Rev. D 78, 025011 (2008); R Ruffini, G. Vereshchagin, S-S. Xue, Phys. Rept. 487, 1 (2010).

18. G. V. Dunne and C. Schubert, Phys. Rev. D 72, 105004 (2005); G. V. Dunne, Q.H. Wang, H. Gies, and C. Schubert, Phys. Rev. D 73, 065028 (2006).

19. W. Pauli, Rev. Mod. Phys. 13, 203 (1941).

20. P. M. Lavrov, J. Phys. A 18, 3455 (1985); W. Dittrich and M. Reuter, Lect. Notes Phys. 220, 1 (1985); C.-L. Ho and P. Roy, Ann. Phys. 312, 161 (2004); Q.-G. Lin, Phys. Rev. A 61, 022101 (2000).

21. S. P. Kim, H. K. Lee, and Y. Yoon, Phys. Rev. D 79, 045024 (2009); S. P. Kim and H. K. Lee, Phys. Rev. D 76, 125002 (2007).

22. S. P. Kim, H. K. Lee, and Y. Yoon, Phys. Rev. D 82, 025015 (2010).

23. S. P. Kim, H. K. Lee, and Y. Yoon, Phys. Rev. D 82, 025016 (2010). 